\title{
CONDIÇÃO DE SAÚDE BUCAL DE PACIENTES ONCOLÓGICOS
}

\section{ORAL HEALTH CONDITION FOR ONCOLOGICAL PATIENTS}

Bruna Braga da Cruz Melo', Gabriela Brito Vasconcelos ${ }^{2}$, Rafaela Brito Vasconcelos ${ }^{3}$,

Ana Carolina de Souza Leitão Arruda Falcão4, Viviane Colares Soares de Andrade Amorim ${ }^{5}$

1. Cirurgiä-Dentista (FOP-UPE).

2. Cirurgiä-Dentista, Mestranda do Programa de Pós-graduação em Hebiatria (FOP-UPE).

3. Cirurgiä-Dentista, Odontopediatra (CPGO-PE).

4. Cirurgiä-Dentista, Pós Doutoranda em Odontopediatria (FOP-UPE), Doutora em Odontologia Integrada (UFPE).

5. Professora Associada de Odontopediatria da Universidade de Pernambuco (FOP-UPE) e da Universidade Federal de Pernambuco (UFPE).

Palavras-chave:

Crianças; Oncologia; Cárie; Biofilme.

Keywords:

Children; Oncology; Caries; Biofilm.

\begin{abstract}
RESUMO
Este estudo teve como objetivo avaliar a condição de saúde bucal de pacientes em terapia oncológica. Trata-se de um estudo observacional transversal realizado no Centro OncoHematologico de Pernambuco (CEONHPE), vinculado ao Hospital Universitário Oswaldo Cruz (HUOC), da Universidade de Pernambuco (UPE). Foram incluídos no estudo pacientes com idade entre 1 e 19 anos, de ambos os sexos, totalizando uma amostra de 177 pacientes, dentre os quais 25 realizaram o índice de placa, 111 submeteram-se ao índice de Dentes Cariados, Perdidos e Obturados (CPOD) e 129 ao índice de Dentes Decíduos Cariados, Com Extração Indicada e Obturados (ceo-d). Foi realizada coleta de dados secundários obtidos nas fichas clínicas dos pacientes que estiveram em atendimento no ano de 2018. Através da análise estatística descritiva foram consideradas as variáveis: condição de saúde bucal, idade, gênero, diagnóstico oncológico e fase atual do tratamento. Com relação às condições de saúde bucal foram avaliadas: condições de higiene oral (índice de placa de O'Leary); cárie (CPO-D e ceo-d). Verificou-se que houve predominância do índice CPO-D e ceo-d em zero e o índice de placa foi considerado insatisfatório na maioria dos pacientes. Portanto, apesar de baixo índice de cárie, foi constatado elevado índice de placa bacteriana.
\end{abstract}

\section{ABSTRACT}

This study aimed to assess the oral health condition of patients undergoing cancer therapy. This is a cross-sectional observational study carried out at the Centro OncoHematologico de Pernambuco (CEONHPE), linked to the Hospital Universitário Oswaldo Cruz (HUOC), of the University of Pernambuco (UPE). The study included patients aged between 1 and 19 years old, of both sexes, totaling a sample of 177 patients, among whom 25 underwent the plaque index, 111 underwent the Decayed, Lost and Filled Teeth Index (CPOD) and 129 to the Decayed Deciduous Teeth Index, with Extraction Indicated and Filled (ceo-d). Secondary data were collected from the clinical records of patients who were in care in 2018. Through the descriptive statistical analysis, the variables were considered: oral health condition, age, gender, cancer diagnosis and current treatment stage. Regarding oral health conditions, the following were evaluated: oral hygiene conditions (O'Leary plaque index); caries (CPOD e ceo-d). It was found that there was a predominance of the CPOD index and ceo- $d$ at zero and the plaque index was considered unsatisfactory in most patients. Therefore, despite a low rate of caries, a high rate of plaque was found.
Autor Correspondente:

Gabriela Brito Vasconcelos

Telefone: (+ 55 81) 988382803

E-mail: gabibvasconcelos@hotmail.com

\section{INTRODUÇÃO}

As neoplasias malignas em crianças e adolescentes devem ser abordadas de forma diferente de suas manifestações em adultos, dada a diferença entre os locais primários, a origem histológica e comportamento clínico. Comumente apresenta crescimento rápido, maior capacidade de invasão, contudo, tende a responder melhor ao tratamento ${ }^{1}$.

Cerca de $70 \%$ das crianças diagnosticadas precocemente tem chance de cura. $\mathrm{O}$ tratamento consiste em cirurgia, quimioterapia, radioterapia e em alguns casos, transplante de medula óssea².

O tratamento quimioterápico na dependência do seu tipo, dosagem e frequência, bem como a radioterapia; podem trazer uma série de complicações ao meio bucal, como resultado, por exemplo, do efeito imunossupressor. As principais alterações são: xerostomia, mucosite, osteorradionecrose e cárie, direta ou indiretamente (efeito tardio). Conflitos internos e desorganização psíquica também podem afetar o paciente durante o curso da doença ${ }^{1-5}$. 
O cuidado com a saúde bucal destes pacientes se faz essencial e depende de ações distintas e simultâneas: do apoio familiar, da motivação individual, de políticas públicas específicas, além da abordagem profissional multidisciplinar. A odontopediatra tem papel importante nas diversas etapas do tratamento oncológico, desde abordagens preventivas até abordagens reabilitadoras, o que vai colaborar efetivamente para uma melhor qualidade de vida destes indivíduos em terapia ${ }^{6,7}$.

Desta forma, esse estudo teve como objetivo avaliar a condição de saúde bucal de pacientes oncológicos, atendidos no CEONHPE, vinculado ao HUOC, da UPE. A avaliação da situação de saúde bucal desse grupo específico, contribui na definição de protocolos de atendimento e políticas públicas mais eficientes.

\section{MATERIAIS E MÉTODOS}

Trata-se de um estudo transversal, observacional, de caráter descritivo, realizado com crianças, adolescentes e jovens sob tratamento oncológico, por meio da análise dos dados coletados nas fichas clínicas médicas e odontológicas. O estudo foi realizado em Recife, Pernambuco, no CEONHPE, vinculado ao Hospital HUOC da UPE. Como população-alvo, foram considerados crianças e adolescentes de 1 a 19 anos, de ambos os sexos, que estiveram em tratamento no ano de 2018. A amostra foi composta pelos pacientes atendidos no consultório odontológico do CEONHPE pelo grupo de estudantes de graduação de odontologia, sob supervisão de uma professora de Odontopediatria, como atividade do Projeto de Extensão Fazendo ART com as crianças. Foram excluídos da pesquisa, os pacientes que por algum comprometimento cognitivo não puderam responder à anamnese e/ou que seus responsáveis não estavam presentes no momento do exame.

Foram anotados dados a respeito da idade, gênero, diagnóstico oncológico e fase atual do tratamento oncológico do paciente, assim como o índice CPOD e índice de placa de O'leary. Para coleta de dados de exame clínico, foram registrados sobre as condições de higiene oral e condições quanto á cárie.

Durante o exame clínico odontológico, a fim de avaliar a condição de higiene bucal, foi avaliado o índice de Placa Bacteriana. Foi aplicado o índice de Placa O'Leary para mensuração da porcentagem de placa bacteriana, através de solução evidenciadora de placa Visuplac (Maquira Solução $10 \mathrm{ml}$ ) aplicada nas quatro faces de todos os dentes presentes na arcada, facilitando ao paciente a visualização das possíveis falhas na escovação. O cálculo dá-se pela divisão do número de faces coradas multiplicado por cem pelo número de dentes presentes multiplicado por quatro. Por fim, estabelece-se para critérios de nível de higienização bucal: de $0 \%$ até $25 \%$ como satisfatório, de $25 \%$ a $40 \%$ como irregular e de $40 \%$ acima é tido como insatisfatório ${ }^{8}$. Pacientes em tratamento clínico não realizaram a evidenciação de placa por não apresentarem condições sistêmicas favoráveis e risco de sangramento.

Para fins de diagnóstico da doença Cárie, foram considerados os índices CPO-D para os pacientes que se encontravam na dentição mista ou permanente, utilizado pela Organização Mundial de Saúde (OMS) para avaliar a prevalência de cárie e índice ceo-d para os pacientes que apresentavam dentição decídua. $O$ registro foi realizado após a inspeção visual dos dentes presentes. Pacientes com impossibilidades sistêmicas devido ao tratamento não realizaram os índices.

Os dados secundários, sobre informações do tratamento oncológico, foram coletados dos prontuários hospitalares. Foi identificado o tipo de câncer diagnosticado em cada paciente, assim como categorizados os momentos do tratamento que o paciente se encontrava: em pré tratamento (1), em tratamento clínico (2), em tratamento radioterápico (3), em tratamento quimioterápico (4), em remissão (5).

Os dados foram analisados através da estatística descritiva e utilizados em números absolutos e percentuais. Os resultados foram apresentados na forma de tabelas e gráficos.

A pesquisa teve aprovação do Comitê de Ética em Pesquisa da UPE, Certificado de Apresentação de Apreciação Ética (CAAE) 03501218.9.0000.5207 bem como foi conduzido de acordo com o estabelecido pela Resolução número 196/96 do Conselho Nacional de Saúde, do Ministério da Saúde, para experimentos em humanos.

\section{RESULTADOS}

A amostra desse estudo foi formada por 177 pacientes, em sua maioria do sexo masculino $(61,67 \%)$, sendo $15,56 \%$ menores de 3 anos, 20,5\% de 4 a 6 anos, 19,44\% de 7 a 9 anos, $26,7 \%$ de 10 a 14 anos e $17,78 \%$ de 15 a 19 anos. O índice de placa foi realizado em 25 pacientes (Figura 1).

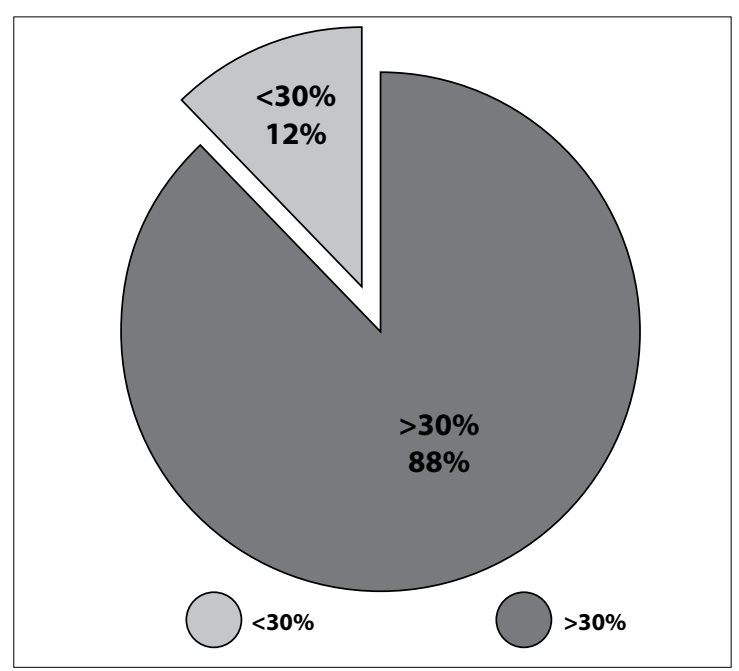

Figura 1 - Distribuição dos pacientes de acordo com o índice de placa.

A maioria dos pesquisados apresentou CPO-D e ceo-d igual a zero (Tabela 1) (Tabela 2). A maioria dos pacientes encontravam-se em remissão, seguindo da fase de pré tratamento (Tabela 3 ). 
Saúde bucal de pacientes oncológicos

Melo BBC, et al.

Tabela 1 - Distribuição dos pacientes de acordo com o CPO-D.

\begin{tabular}{lcc}
\hline \multicolumn{1}{c}{ Variáveis } & n & $\%$ \\
\hline CPOD & 62 & 55,9 \\
0 & 32 & 28,8 \\
1 a 3 & 16 & 14,4 \\
$\geq 4$ & & \\
cariados (C) & 82 & 73,9 \\
0 & 22 & 19,8 \\
1 a 3 & 6 & 05,4 \\
$\geq 4$ & & \\
Restaurados (o) & 82 & 73,9 \\
0 & 25 & 22,5 \\
1 a 3 & 4 & 03,6 \\
$\geq 4$ & & \\
Perdidos (p) & 100 & 90,1 \\
0 & 10 & 09,0 \\
1 a 3 & 1 & 01,0 \\
$\geq 4$ & $\mathbf{1 1 1}$ & $\mathbf{1 0 0 , 0}$ \\
\hline Total & & \\
\hline
\end{tabular}

Tabela 2 - Distribuição dos pacientes de acordo com o ceo-d.

\begin{tabular}{|c|c|c|}
\hline Variáveis & $\mathbf{n}$ & $\%$ \\
\hline \multicolumn{3}{|l|}{ Ceo-d } \\
\hline 0 & 85 & 65,90 \\
\hline $1 \mathrm{~A} 3$ & 28 & 21,80 \\
\hline$\geq 4$ & 16 & 12,40 \\
\hline
\end{tabular}

cariados (c)

72,87

1 a $3 \quad 24 \quad 18,60$

$\geq 4 \quad 11 \quad 8,52$

Restaurados (o)

0

1 a 3

91,48

7,76

0,78

Perdidos (p)

0

1 a 3

96,90

\begin{tabular}{lcc}
$\geq 4$ & 1 & 0,78 \\
\hline Total & 129 & 100
\end{tabular}

Tabela 3 - Distribuição de pacientes de acordo com a fase do tratamento e o índice CPOD.

\begin{tabular}{|c|c|c|c|c|c|c|c|c|}
\hline & \multirow{2}{*}{\multicolumn{2}{|c|}{ Total }} & \multicolumn{6}{|c|}{ CPO-D } \\
\hline & & & \multicolumn{2}{|c|}{0} & \multicolumn{2}{|c|}{1 a 3} & \multicolumn{2}{|c|}{$\geq 4$} \\
\hline & $\mathbf{N}$ & $\%$ & $\mathbf{N}$ & $\%$ & $\mathbf{N}$ & $\%$ & $\mathbf{N}$ & $\%$ \\
\hline Não iniciou o tratamento & 23 & 20,72 & 14 & 6,93 & 6 & 5,40 & 3 & 2,70 \\
\hline Radioterapia & 1 & 1,00 & 1 & 0,50 & 0 & 0 & 0 & 0 \\
\hline Quimioterapia & 13 & 11,71 & 4 & 3,60 & 4 & 3,60 & 5 & 4,50 \\
\hline Remissão & 74 & 66,67 & 42 & 37,84 & 22 & 19,81 & 10 & 9,00 \\
\hline Total & 111 & 100 & 61 & 54,96 & 32 & 28,82 & 18 & 16,21 \\
\hline
\end{tabular}

O índice CPOD igual a zero aparece em maior quantidade em todos os diagnósticos (Leucemia, linfoma, doença do SNC, sem diagnóstico), havendo uma leve tendência do índice igual a quatro ou mais em outros tipos de tumores.

Os pacientes que se encontram na fase de pré tratamento e remissão obtiveram em sua maioria um índice de placa de O'leary insatisfatório.

O índice de placa apresenta-se elevado em todos os tipos de tumores, exceto nos linfomas. Em pacientes portadores de leucemias, outros tumores e sem diagnóstico o índice de placa foi elevado nas suas maiorias (Gráfico 1).

$O$ índice ceo-d igual a zero apresenta-se em maior quantidade quando relacionado a fase do tratamento. Em todas as fases nota-se sua expressiva repetição (Tabela 4).

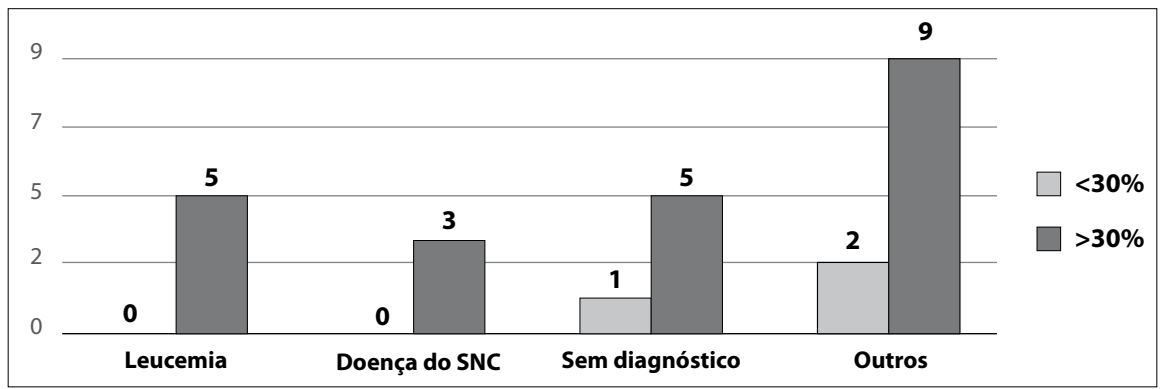

Gráfico 1 - Distribuição de pacientes de acordo com o diagnóstico e o índice de placa O'leary. 
Saúde bucal de pacientes oncológicos Melo BBC, et al.

Tabela 4 - Distribuição de pacientes de acordo com a fase de tratamento e o Ceo-d.

\begin{tabular}{|c|c|c|c|c|c|c|c|c|}
\hline & \multirow{2}{*}{\multicolumn{2}{|c|}{ Total }} & \multicolumn{6}{|c|}{ Ceo-d } \\
\hline & & & \multicolumn{2}{|c|}{0} & \multicolumn{2}{|c|}{1 a 3} & \multicolumn{2}{|c|}{$\geq 4$} \\
\hline & $\mathbf{N}$ & $\%$ & $\mathbf{N}$ & $\%$ & $\mathbf{N}$ & $\%$ & $\mathbf{N}$ & $\%$ \\
\hline Não iniciou o tratamento & 42 & 32,56 & 29 & 22,49 & 8 & 6,2 & 5 & 3,88 \\
\hline Tratamento clínico & 2 & 1,56 & 1 & 0,78 & 0 & 0 & 1 & 0,78 \\
\hline Radioterapia & 6 & 4,66 & 3 & 2,32 & 3 & 2,32 & 0 & 0 \\
\hline Quimioterapia & 21 & 16,28 & 11 & 8,52 & 4 & 3,1 & 6 & 4,64 \\
\hline Remissão & 58 & 44,97 & 38 & 29,46 & 13 & 10,08 & 7 & 5,42 \\
\hline Total & 129 & 100 & 82 & 63,57 & 28 & 21,70 & 19 & 14,72 \\
\hline
\end{tabular}

Da mesma forma, foi observado que o índice ceo-d igual a zero apresenta-se em maior quantidade quando relacionado ao diagnóstico oncológico.

\section{DISCUSSÃO}

O número de casos de câncer tem aumentado consideravelmente em todo o mundo. Na infância, a incidência de neoplasias malignas varia de 1 a $4 \%$ nos registros de câncer de base populacional ${ }^{9}$. Neste estudo, observa-se que a maioria dos pacientes em terapia oncológica encontram-se entre 10 e 14 anos, configurando crianças e pré-adolescentes como a maioria diagnósticada. Ratificando assim, o que é citado pela literatura em relação à infância e diferenciando-se quando se trata de pré adolescentes.

Os tumores mais comumente encontrados são leucemia e linfomas. Outros tipos também podem estar presentes como neoplasias retículo- endoteliais, tumores de sistema nervoso central e miscelânia de neoplasias intracranianas e intraespinais ${ }^{10}$. Sendo, portanto, de acordo com o presente estudo, o qual foi observado um maior numéro de diagnósticos de leucemia.

O tratamento consiste em cirurgia, radioterapia e/ou quimioterapia. Sua escolha irá depender do tipo e estágio do tumor. As terapias preconizadas podem provocar efeitos colaterais, principalmente devido ao quadro de imunossupressão ${ }^{10}$. Os pacientes tratados com radioterapia são susceptíveis a vários efeitos secundários que incluem principalmente mucosite, perda do paladar, xerostomia e trismo ${ }^{11,12}$. Já a quimioterapia pode favorecer a imunossupressão, levando a lesões na cavidade oral, devido à alta sensibilidade dos tecidos e das estruturas bucais aos efeitos tóxicos dos quimioterápicos, o que poderá resultar em complicações sistêmicas importantes ${ }^{3}$. Os dados obtidos nesse estudo nos mostram que a maioria dos pacientes se econtram em cuidados quimioterápicos

O diagnóstico de câncer é vivido como um momento de angústia e ansiedade, pelo motivo da doença ser rotulada como dolorosa e mortal, conseqüentemente, desencadeando preocupações em relação à morte. Além do momento do diagnóstico, ao longo do tratamento, o paciente vivencia perdas e diversos sintomas que, além de acarretar prejuízos ao organismo, incluindo a saúde bucal ${ }^{1,13,14}$. Com relação a cárie dentária, os resultados encontrados neste estudo foram baixos, com CPOD de 1,15 e ceo-d de 1,08. Já o índice de placa foi considerado elevado e insatisfatório, visto que foi concentrado em $>30$.

Os dados obtidos em estudo realizado em Natal$\mathrm{RN}^{15}$ são CPO-D de 0,6 e ceo-d de 1,6 num grupo de pacientes oncológicos, assemelhando-se aos desta pesquisa. $\mathrm{O}$ autor justifica esses índices de cárie baixos devido o fato de que no hospital oncológico onde as crianças foram examinadas ocorram frequentemente programas educativos e preventivos de saúde bucal ${ }^{15}$. Em um estudo realizado em São Paulo ${ }^{16} \mathrm{com}$ pacientes oncológicos e utilizando o índice CPOD, obteve uma média de 24,42 do índice, diferenciando-se bastante da média registrada no presente estudo $(1,15)^{16}$. Já numa pesquisa em Criciuma ${ }^{17}$, percebeu-se que apenas em $26,1 \%$ dos 26 pacientes participantes foi realizada uma consulta odontológica, explicando a condição bucal insatisfatória em grande parte dos estudos ${ }^{17}$. (Os dados obtidos nesse estudo quanto a cárie são consideravelmente menores e os dados obtidos quanto ao índice de placa são semelhantes se comparados aos encontrados por Figueiredo e Nogueira ${ }^{15}$, que avaliando clinicamente a prevalência de cárie e gengivite em 32 crianças portadores de neoplasias, obtiveram uma média do índice CPO-D $=2,166$ e ceo- $d=2,814$ e CPO-D = 2,625 e ceo- $d=3,259$ e biofilme bucal elevado. Na Bélgica, em 1995, foi realizado uma pesquisa que, assim como neste presente trabalho, não encontraram resultados elevados com relação à saúde gengival e higiene oral no grupo de pacientes oncológicos ${ }^{15}$.

A partir disso, o tratamento odontológico previamente ao tratamento oncológico objetiva a eliminação e estabilização de condições orais que desencadeiem uma infecção local ou sistêmica. Para isso, o dentista deve não só saber das indicações odontológicas, mas também do processo de diagnóstico do câncer, estadiamento e planejamento da oncoterapia. Além da atuação no paciente antes do inicio do tratamento, cabe ao cirurgião dentista o acompanhamento em longo prazo desse paciente a fim de evitar complicações bucais crônicas decorrentes do tratamento oncológico. Portanto, a função do dentista é atuar junto à equipe médica para prevenir e tratar complicações bucais decorrentes do tratamento antineoplásico ${ }^{16,18}$. 


\section{CONCLUSÃO}

Concluiu-se que a maioria dos pacientes dessa amostra apresentaram baixo índice de cárie, sendo 62 e 85 indivíduos com CPOD e ceo- $d=0,32$ e 28 apresentando CPOD e ceo- $d$ entre 1 e 3 , respectivamente, 16 com CPOD e ceo- $d>4$. O índice de placa foi elevado na maioria dos pacientes que foi realizado.

\section{FINANCIAMENTO}

A pesquisa não recebeu financiamento para sua realização.

\section{CONFLITO DE INTERESSES}

Não houve conflitos de interesses

\section{AGRADECIMENTOS}

Agradecemos a colaboração e atenção de todas crianças e adolescentes atendidos durante a pesquisa, no CEONHPE do HUOC da Universidade de Pernambuco.

\section{REFERÊNCIAS}

1. Silva S, Aquino T, Santos R. O paciente com câncer: cognições e emoções a partir do diagnóstico. Rev Bras Ter Cogn. 2008;4(2):73-88.

2. Lopes LF, Camargo B, Bianchi A. Os efeitos tardios do tratamento do câncer infantil. Rev Assoc Med Bras. 2000;46(3):277-84.

3. Hespanhol FL, Tinoco E, Teixeira H, Falabella M, Assis N. Manifestações bucais em pacientes submetidos à quimioterapia. Ciênc saúde coletiva. 2010;15(Supl 1):1085-94.

4. Grimaldi N, Sarmento V, Provedel L, Almeida D, Cunha S. Conduta do cirurgião-dentista na prevenção e tratamento da osteorradionecrose: revisão de literatura. Rev Bras Cancerol. 2005;51(4):319-24.

5. Santos CC, Noro-Filho GA, Caputo BV, Souza RC, Andrade DM, Giovani EM. Condutas práticas e efetivas recomendadas ao cirurgião dentista no tratamento pré, trans e pós do câncer bucal. J Health Sci Inst. 2013;31(4):368-72.

6. Goursand D, Borges CM, Alves KM, Nascimento AM, Winter RR, Martins LHPM, et al. Sequelas bucais em crianças submetidas a terapia antineoplasica: causas e definicção do papel do cirurgião dentista. Arq Odontol. 2016;42(3):180-9.

7. Anais da VI Mostra Interna de Trabalhos de Iniciação Científica; 2012 Out 23-26; Maringá, PR. Maringá: CESUMAR, 2012.

8. Silva A, Barbosa B, Batista B, Reis C, Ruas C, Ferreira C, et al. Análise do Índice de O'Leary [Internet]. [place unknown]: Prezi, c2020 [cited 2020 May 20]. Available from: https://prezi. com/5qlmrouidtn5/analise-do-indice-de-o039leary/.

9. Beltrão $M$, Vasconcelos $M$, Pontes $C$, Albuquerque $M$. Childhood cancer: maternal perceptions and strategies for coping with diagnosis. J Pediatr. 2007; 3(6):562-5.
10. 10. Frazão C, Alfaya T, Costa R, Rocha $M$, Gouvêa C, Morais A. Pacientes oncológicos pediatricos: manifestações bucais da terapia antineoplasica. Saude e Pesqui. 2012;5(3):587-92. 11. Peres $P$, Queiroz AM, Moreira MR, Faquim JPS, Ferrari MA. Odontopediatria aplicada ao câncer infantil - manifestações clínicas e protocolos de atendimento. J Manag Prim Health Care. 2013;4(3):191-9.

12. Gaetti-Jardim Júnior E, Souza F, Gaetti-Jardim E, Castro E, Ciesielski F, Buso-Ramos M, et al. Efeitos da radioterapia sobre as condições bucais de pacientes oncológicos. RPG Rev Pós Grad. 2011;18(2):96-101.

13. Cavicchioli A, Menossi M, Lima R. Câncer infantil: o intinerário diagnóstico. Rev Latino-Am Enfermagem. 2007;15(5):1025-32.

14. Faria MT. Atendimento odontológico ao paciente com câncer: orientação para cirrugiões dentistas [dissertation]. Volta Redonda, RJ: Fundação Oswaldo Aranha; 2017;71.

15. Figueiredo PBA, Nogueira AJS. Prevalência de Neoplasias, Cárie e Gengivite em Pacientes Oncológicos Pediátricos no Município de Belém, Pará. Pesq Bras Odontoped Clin Integr. 2013;13(2):141-6.

16. Quispe R, Cremonesi A, Gonçalves J, Rubira C, Santos P. Estudo caso-controle de índices de doenças bucais em indivíduos com câncer de cabeça e pescoço após terapia antineoplásica. Einstein. 2018;16(3):1-6.

17. Rosso $M$, Neves $M$, Araújo $P$, Ceretta $L$, Simões $P$, Sõnego $F$, et al. Análise da condição bucal de pacientes pediatricos e adolescentes portadores de neoplasias na instituição casa guido na cidade de criciúma. Rev Odontol Univ Cid São Paulo. 2015;27(3):210-9.

18. Silveira J, Oliveira V, Padilha W. Avaliação da redução do índice de placa visível e do índice de sangramento gengival em uma prática de promoção de saúde bucal com crianças. Pesqui Odontol Bras. 2002;16(12):169-174. 